\title{
Sex differences in glucose metabolism of streptozotocin-induced diabetes inbred mice (C57BL/6J)
}

Boyoung Kim', Yoo Yeon Kim', Phuong Thi-Thanh Nguyen ${ }^{1}$, Hajin Nam ${ }^{1}$ and Jun Gyo Suh ${ }^{1,2^{*}}$ (D)

\begin{abstract}
Considering that sex differences in glucose metabolism are observed in mice, researchers unconsciously use male mice to reduce variations by an estrogen cycle in female mice. In this study, we investigated the sex differences in glucose homeostasis in streptozotocin (STZ)-induced diabetes inbred mice (C57BL/6J). The C57BL/6J male and female mice were injected with or without STZ (40 mg/kg) for 5 consecutive days. Levels of fasting blood glucose (FBG), glycosylated hemoglobin $\left(\mathrm{HbA}_{1 \mathrm{C}}\right)$, lipid profiles, oral glucose tolerance, and insulin resistance were measured at 3 and 6 weeks after STZ treatment. The FBG level in the STZ-induced male (M-STZ) group was significantly higher than that in the STZ-induced female (F-STZ) group during the entire experimental period. Furthermore, $\mathrm{HbA}_{1 \mathrm{C}}$ and glucose tolerance levels in the M-STZ group were significantly higher than those in the F-STZ group at 3 and 6 weeks after STZ treatment. The glucagon/insulin ratio in the M-STZ group was significantly higher than that in the F-STZ group. Values of the homeostatic model assessment-insulin resistance, an indicator of $\beta$-cell function and insulin resistance, significantly increased in both the M-STZ and F-STZ groups at 3 weeks after STZ treatment. However, insulin resistance was observed in the M-STZ group, but not in the F-STZ group, at 6 weeks after STZ treatment. Taken together, our results indicate that glucose metabolism in the M-STZ group was worse than that in the F-STZ group, indicating that estrogen may have an important role in glucose metabolism by STZ treatment.
\end{abstract}

Keywords: Blood glucose, Diabetes, Glucose tolerance, Sex differences, Streptozotocin

\section{Introduction}

Researchers prefer to use male animals to reduce variations in their experimental results. Researchers believe that animal experiment results influence the estrogen cycle of female animals. Certainly, male animals are used 5.5 times more compared to female animals in neuroscience and biomedical researches [1]. There are several reports stating that sex is an important factor affecting sex differences in animals and humans. The adiposity of mice was influenced by the number of $\mathrm{X}$ chromosomes. The adiposity of female (XX chromosomes) mice was

\footnotetext{
*Correspondence: jgsuh@hallym.ac.kr

${ }^{1}$ Department of Medical Genetics, College of Medicine, Hallym University,

1, Hallymdaehak-gil, Chuncheon, Gangwon-do 24252, South Korea

Full list of author information is available at the end of the article
}

twofold higher than that of male (XY chromosomes) mice under the same conditions [2]. The pain of mice and rat inhibitors by male experimenters (but not female) indicates that experimenter sex is the most important factor in pain research [3]. Chronic pain hypersensitivity in male mice is induced by microglia-to-neuron signaling. However, that of female mice is induced by adaptive immune cells, not microglia [4]. In humans, the preventive effects of low-dose aspirin are different in women and men. Zolpidem, a drug for insomnia, also uses different doses in women and men. Considering these reasons, the National Institute of Health recommends that grant applicants must consider sex differences in their experimental plan [5].

The incidence of diabetes mellitus is significantly increasing worldwide. Thus, it is considered a public 
health issue with an enormous cost of medical treatment. Glucose metabolism and diabetes mellitus differ between men and women [6]. Insulin resistance (IR) in women is more increased compared to that in men because women have higher adipose and lower skeletal muscle mass than men of the same age [7]. Early menopause is an important risk factor for type 2 diabetes $[8,9]$, and ovariectomy also increases the risk of acquiring diabetes [10]. Prostate cancer patients treated with androgen depletion therapy are more susceptible to diabetes compared to prostate cancer patients not treated with androgen depletion therapy [11]. The diastolic dysfunction of streptozotocin (STZ)-induced diabetic female mice was more severe than that of male mice [12]. Diabetic Torii Lepr ${ }^{\text {fa }}$ rats have sex differences in blood glucose and insulin levels. Diabetic symptoms in male rats are observed earlier and are more critical compared to female rats [13].

Despite the differences in glucose metabolism and diabetes in men and women, researchers have used only male animal models to study the mechanisms of diabetes and to develop antidiabetic drugs. This is one of the main reasons for drug development failure in diabetes. To understand the detailed sex differences in mice, we investigated the sex differences in glucose metabolism in STZ-induced diabetes C57BL/6J mice. The levels of fasting blood glucose (FBG) and glucose tolerance in males were significantly compared to those in females, indicating that estrogen may have an important role in glucose metabolism after STZ treatment.

\section{Materials and methods}

\section{Animals and streptozotocin (STZ) treatment}

Eight-week-old C57BL/6J mice were obtained from the DBL Company (Korea). The mice were divided into the following four groups: (1) the C57BL/6J male control (M-con, $\mathrm{n}=10)$, (2) STZ-induced male (M-STZ, $\mathrm{n}=10$ ), (3) C57BL/6J female control (F-con, $\mathrm{n}=10$ ), and (4) STZ-induced female (F-STZ, $\mathrm{n}=10$ ) groups. Mice were treated with STZ $(40 \mathrm{mg} / \mathrm{kg})$ in $50 \mathrm{mM}$ citrate buffer $(\mathrm{pH}, 4.5)$ for 5 consecutive days via the intraperitoneal route. To prevent fatal hypoglycemia, mice freely received $10 \%$ sucrose water from 1 to 6 days after STZ treatment. The animal facility maintained an environment temperature of $22 \pm 2{ }^{\circ} \mathrm{C}$, a humidity of $55 \pm 10 \%$, and a 12-h light cycle (8 A.M. -8 P.M.) with normal experimental mice chow diet (Purina, Korea) and water ad libitum. The animal experiments were conducted according to the guidelines of the "Institutional Animal Care and Use Committee" of Hallym University (Hallym2018-55).

\section{Measurement of fasting blood glucose, hemoglobin $A_{1 C}$, and lipid profile levels}

Mice were fasted for $6 \mathrm{~h}$, and subsequently, blood was collected from the retro-orbital plexus using a capillary tube. FBG level was measured using a blood glucose monitoring meter (Accu-Chek, Roche, USA). Glycosylated hemoglobin $\left(\mathrm{HbA}_{1 \mathrm{C}}\right)$ level was measured using whole blood with an $\mathrm{HbA}_{1 \mathrm{C}}$ analyzer $\left(\right.$ careSURE $^{\mathrm{TM}}$ Analyzer 100, Wellsbio, Korea). Lipid profile levels were measured as previously described [14].

\section{Oral glucose tolerance test}

An oral glucose tolerance test (OGTT) was performed at 3 and 6 weeks after STZ treatment. All groups were fasted for $6 \mathrm{~h}$. Mice were orally administered with $2 \mathrm{~g} /$ $\mathrm{kg}$ of D-glucose (Sigma, USA). Blood glucose levels were measured at $0,30,60$, and $120 \mathrm{~min}$ after $\mathrm{D}$-glucose administration. To determine whether glucose tolerance was observed, the area under the curve (AUC) was calculated.

\section{Histological analysis}

Mice were anesthetized with isoflurane and transcardially perfused with $4 \%$ paraformaldehyde. The dissected pancreas was fixed in $4 \%$ paraformaldehyde, embedded in paraffin, and cut into $5-\mu \mathrm{m}$ sections with an automatic rotary microtome (Reichert-Jung, Germany). To reduce nonspecific hybridization, sections were washed with $1 \mathrm{X}$ Tris-buffered saline for $5 \mathrm{~min}$ followed by blocking buffer for $30 \mathrm{~min}$. Insulin expression in islets was detected with an anti-insulin antibody (1:1000, Santa Cruz Biotechnology Inc., USA) and donkey anti-rabbit immunoglobulin G (IgG) secondary antibody (1:1000, Abcam, UK). Glucagon expression was observed with donkey anti-goat IgG secondary antibody (1:2000, Abcam, UK) following the incubation with anti-glucagon (1:100, Santa Cruz Biotechnology Inc., UAS) antibody. Confocal microscopy (LSM 710, Carl Zeiss, Germany) was performed to capture the images. The expression of insulin and glucagon in the pancreas was measured using an Image J program (NIH, USA) with at least three mice per group.

\section{Homeostatic model assessment-insulin resistance (IR)}

To assess $\beta$-cell function and IR, the public homeostatic model assessment (HOMA) 2 calculator was used in this study (https://www.dtu.ox.ac.uk/homacalcul ator/). HOMA-IR was calculated according to the following formula: fasting insulin $(\mu \mathrm{U} / \mathrm{L}) \times$ fasting glucose $(\mathrm{nmol} / \mathrm{L}) / 22.5$. 


\section{Statistical analyses}

Results are expressed as the mean \pm standard deviation. The differences between groups were analyzed by one-way analysis of variance using the Statistical Package for the Social Sciences (SPSS) Statistics version 22.0 (SPSS Inc., USA). Statistical significance was defined at $\mathrm{P}<0.05$.

\section{Results}

Fasting blood glucose and glycosylated hemoglobin levels of the STZ-induced male (M-STZ) group were significantly higher than those of the STZ-induced female (F-STZ) group The FBG level of the M-STZ group was significantly higher than that of the M-con group throughout the experimental period (Fig. 1a). The FBG level of the F-STZ group was also significantly higher than that of the F-con group during the entire experimental period (Fig. 1b). There was no significant difference in the FBG levels between the M-con and F-con groups at 6 weeks after STZ treatment $(135.40 \pm 9.50 \mathrm{mg} / \mathrm{dL}$ and $125.40 \pm 15.81 \mathrm{mg} / \mathrm{dL}$, respectively). The FBG levels of the M-STZ and F-STZ groups were $236.20 \pm 26.07 \mathrm{mg} / \mathrm{dL}$ and $157.80 \pm 16.60 \mathrm{mg} / \mathrm{dL}$, respectively, at 6 weeks after STZ treatment. The FBG levels of the F-STZ group were significantly lower than those of the M-STZ group from 1 to 6 weeks after STZ treatment, indicating that female mice are more resistant to STZ toxicity compared to male mice.

$\mathrm{HbA}_{1 \mathrm{C}}$ levels in the M-STZ group were significantly higher than those in the F-STZ group at 3 and 6 weeks after STZ treatment (Fig. 1c). At 6 weeks after STZ treatment, the $\mathrm{HbA}_{1 \mathrm{C}}$ level of the M-STZ group was significantly higher than that of the M-con group (5.32 \pm 0.65 vs. $4.44 \pm 0.11 \%$, respectively, $\mathrm{P}<0.05)$. However, the $\mathrm{HbA}_{1 \mathrm{C}}$ level of the F-STZ group was not significantly different from that of the F-con group at 6 weeks after STZ treatment (Fig. 1c).

\section{Glucose tolerance in the M-STZ group is more severe than that in the F-STZ group}

To determine whether glucose tolerance was observed, an OGTT was performed at 3 and 6 weeks after STZ treatment. At 3 weeks after STZ treatment, FBG levels in the M-STZ and F-STZ groups were significantly higher at 30, 60 , and $120 \mathrm{~min}$ after glucose injection than those of the $\mathrm{M}$-con $(\mathrm{P}<0.01)$ and $\mathrm{F}$-con $(\mathrm{P}<0.01)$ groups, respectively (Fig. 2a). The AUC for blood glucose in the M-STZ group $(1471.50 \pm 102.57)$ was significantly higher than that in the F-STZ group $(1196.20 \pm 52.24, \mathrm{P}<0.01)$, indicating that male mice are more sensitive to STZ treatment than female mice. At 6 weeks after STZ treatment, the AUC for blood glucose in the M-STZ group (1317.40 \pm 175.48$)$
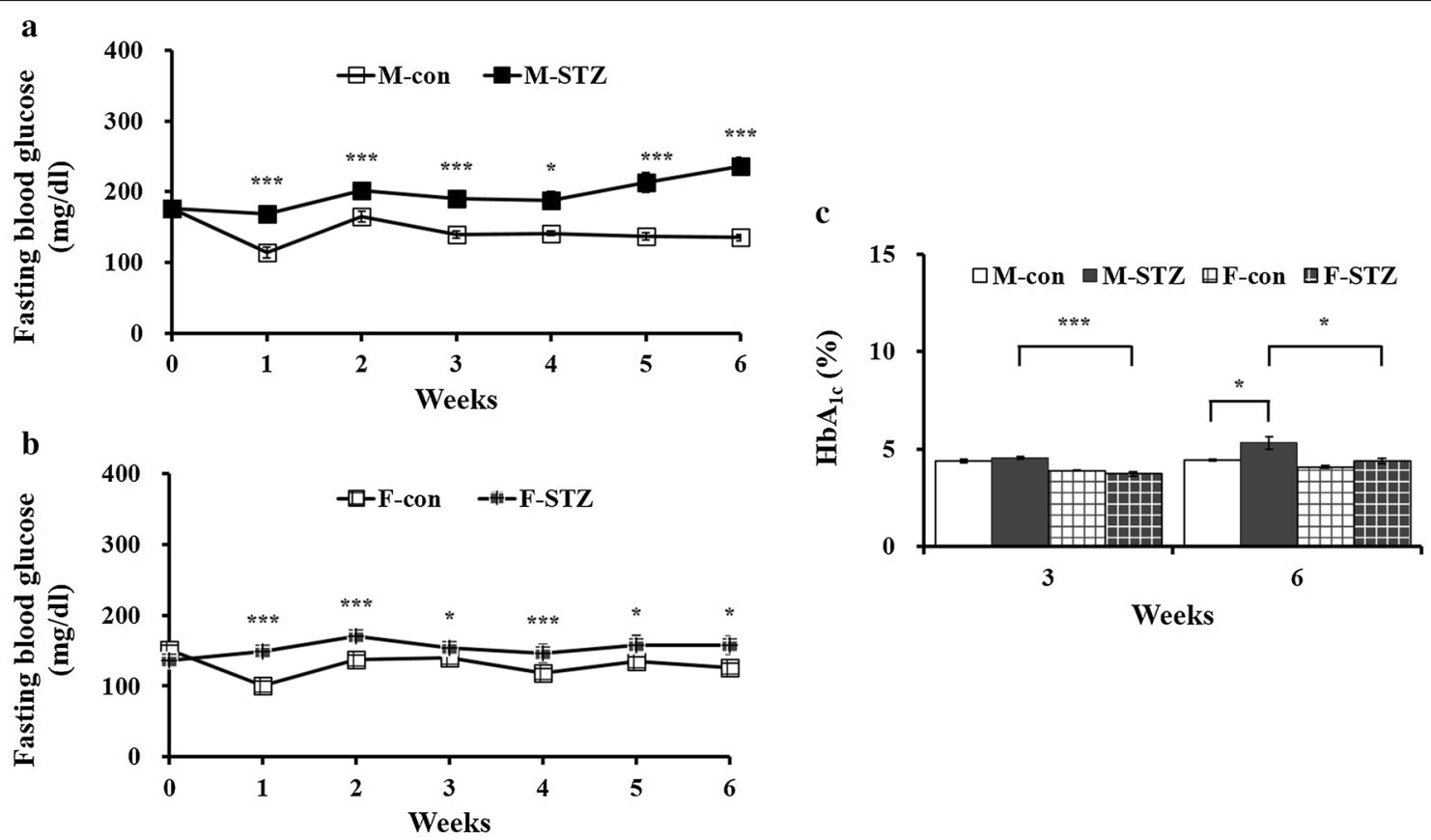

Fig. 1 Sex differences in fasting blood glucose and glycosylated hemoglobin ( $\mathrm{HbA}_{1 \mathrm{C}}$ ) of streptozotocin (STZ)-induced diabetes mice. a Fasting blood glucose level of the STZ-induced male (M-STZ) and male control (M-con) groups. b Fasting blood glucose of the STZ-induced female (F-STZ)

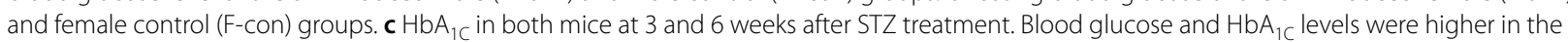
M-STZ group than those in the F-STZ group, suggesting that male mice are more sensitive to STZ toxicity compared to female mice 

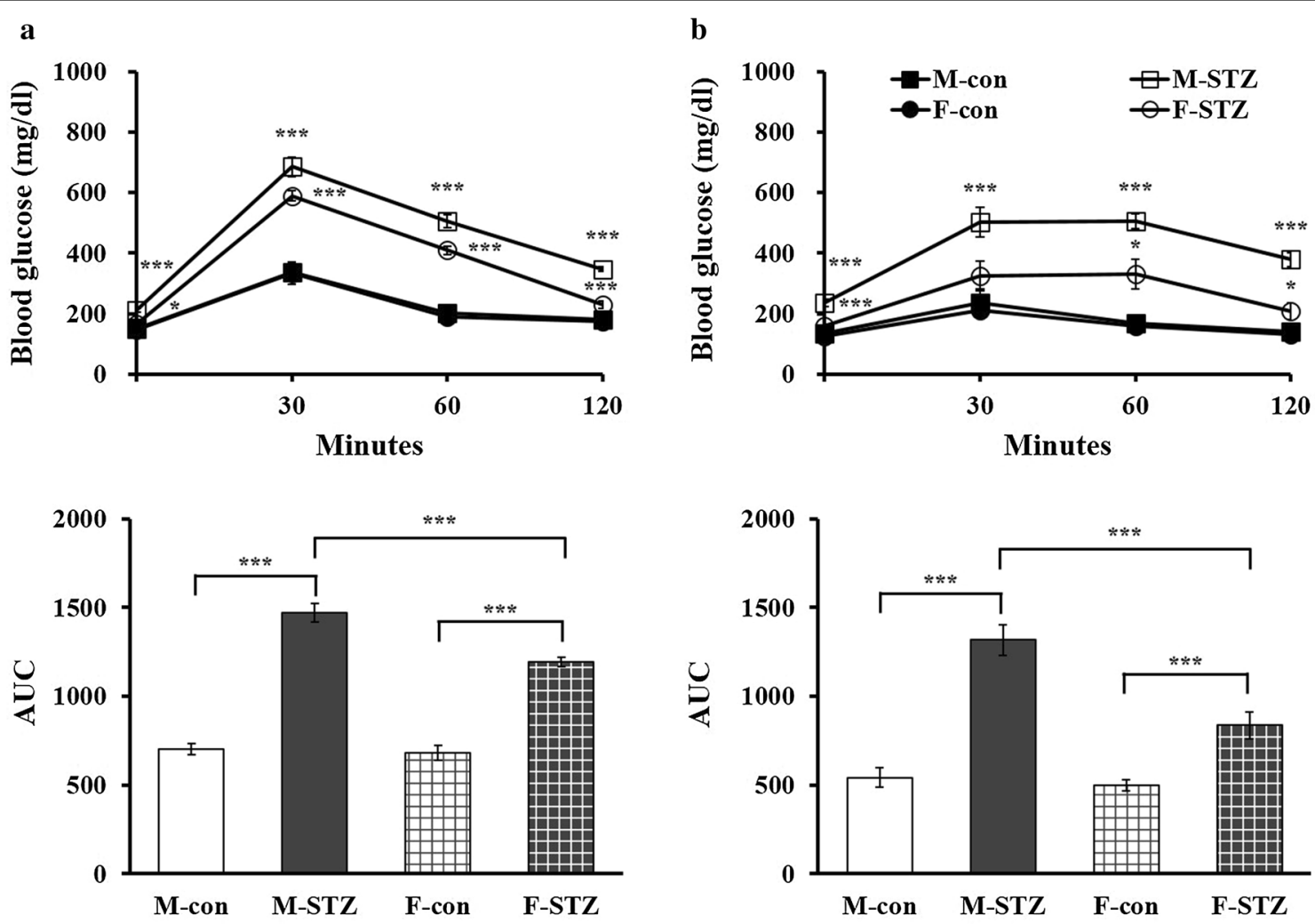

Fig. 2 Sex differences in glucose tolerance in streptozotocin (STZ)-induced diabetes mice. a The area under the curve (AUC) of glucose tolerance at 3 weeks after STZ treatment. b The AUC of glucose tolerance at 6 weeks after STZ treatment. Glucose tolerance in the STZ-induced male group was more severe than that in the STZ-induced female group at 3 and 6 weeks after STZ treatment

was significantly higher than that in the M-con group (541.40 \pm 109.22$)$. The AUC of blood glucose in the M-STZ group was significantly higher than that in the F-STZ group at 6 weeks after STZ treatment (Fig. 2b). The plasma insulin levels of the M-STZ and F-STZ groups were $0.410 \pm 0.03 \mathrm{ng} / \mathrm{mL}$ and $0.410 \pm 0.1 \mathrm{ng} / \mathrm{mL}$ at 6 weeks after STZ treatment, respectively. The plasma insulin levels after STZ treatment were not significantly different compared to each control group.

\section{STZ is more toxic to the pancreatic islets of the M-STZ group compared to those of the F-STZ group}

To verify the morphological changes in pancreatic islets after STZ treatment, $\alpha$ - and $\beta$-cell masses were determined using immunohistochemical analysis. In the $\mathrm{M}$-con and F-con groups, $\beta$ cells, which produce insulin, were localized in the entire region of the pancreatic islets. The $\alpha$ cells, which produce glucagon, were mainly localized in the peripheral region of the pancreatic islets (Fig. 3a).

At 6 weeks after STZ treatment, $\beta$-cell masses in the M-STZ and F-STZ groups were not significantly different compared to that in each control group. The $\beta$-cell mass in the M-STZ group was significantly lower than that in the F-STZ group at the same age (Fig. $3 \mathrm{~b}$ ). The $\alpha$-cell mass in the M-STZ group was significantly higher than that in the M-con group (Fig. 3c). Thus, the glucagon/ insulin ratio in the M-STZ group was significantly different from that in the M-con group (Fig. 3d). The glucagon/ insulin ratio in the M-STZ group was significantly different from that of the F-STZ group at 6 weeks after STZ treatment (Fig. 3d). Taken together, STZ treatment was more toxic in the pancreatic islets in male mice compared to the pancreatic islets in female mice.

\section{The lipid profiles in the F-STZ group are worse than those in the M-STZ group}

The total cholesterol and HDL cholesterol levels significantly increased in the F-STZ and F-con groups at 6 weeks after STZ treatment (Fig. 4a, b). The LDL cholesterol levels in the M-STZ group were significantly higher than those in the M-con group at 3 and 6 weeks after STZ treatment. The LDL cholesterol level in the F-STZ group was also significantly higher than that in the F-con group at 6 weeks after STZ treatment (Fig. 4c). The triglyceride level in the F-STZ group was significantly higher than 
a
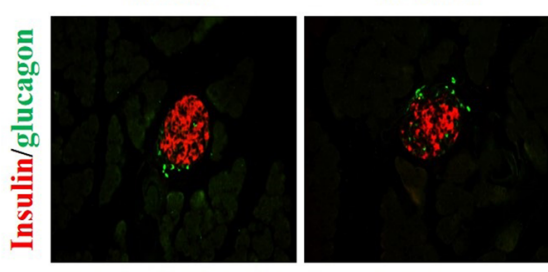

F-con

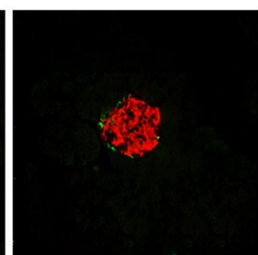

b

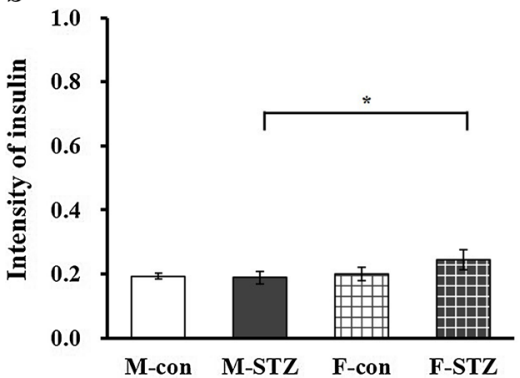

c

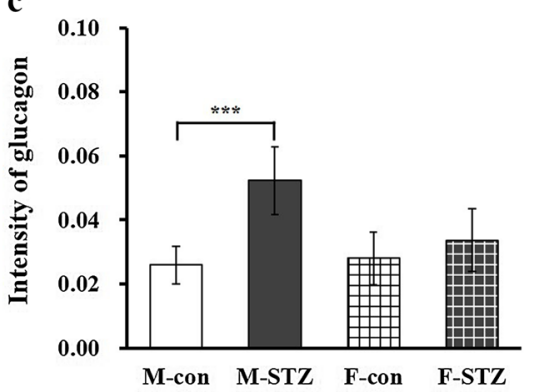

F-STZ

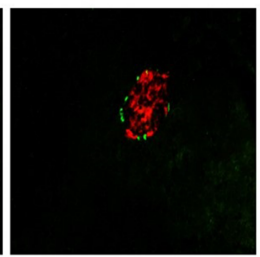

d

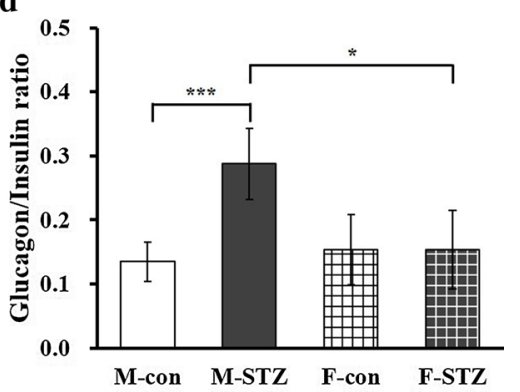

Fig. 3 The $a$ - and $\beta$-cell masses in the pancreatic islets of the streptozotocin (STZ)-induced diabetes mice at 6 weeks after STZ treatment. a Immunostaining with insulin (red) and glucagon (green) antibodies. b $\beta$-cell mass. c a-cell mass. d Glucagon/insulin ratio. The glucagon/insulin ratio in the STZ-induced male group was significantly different from that of the STZ-induced female group, demonstrating that STZ is more toxic in the pancreatic islets of male mice compared to those in female mice
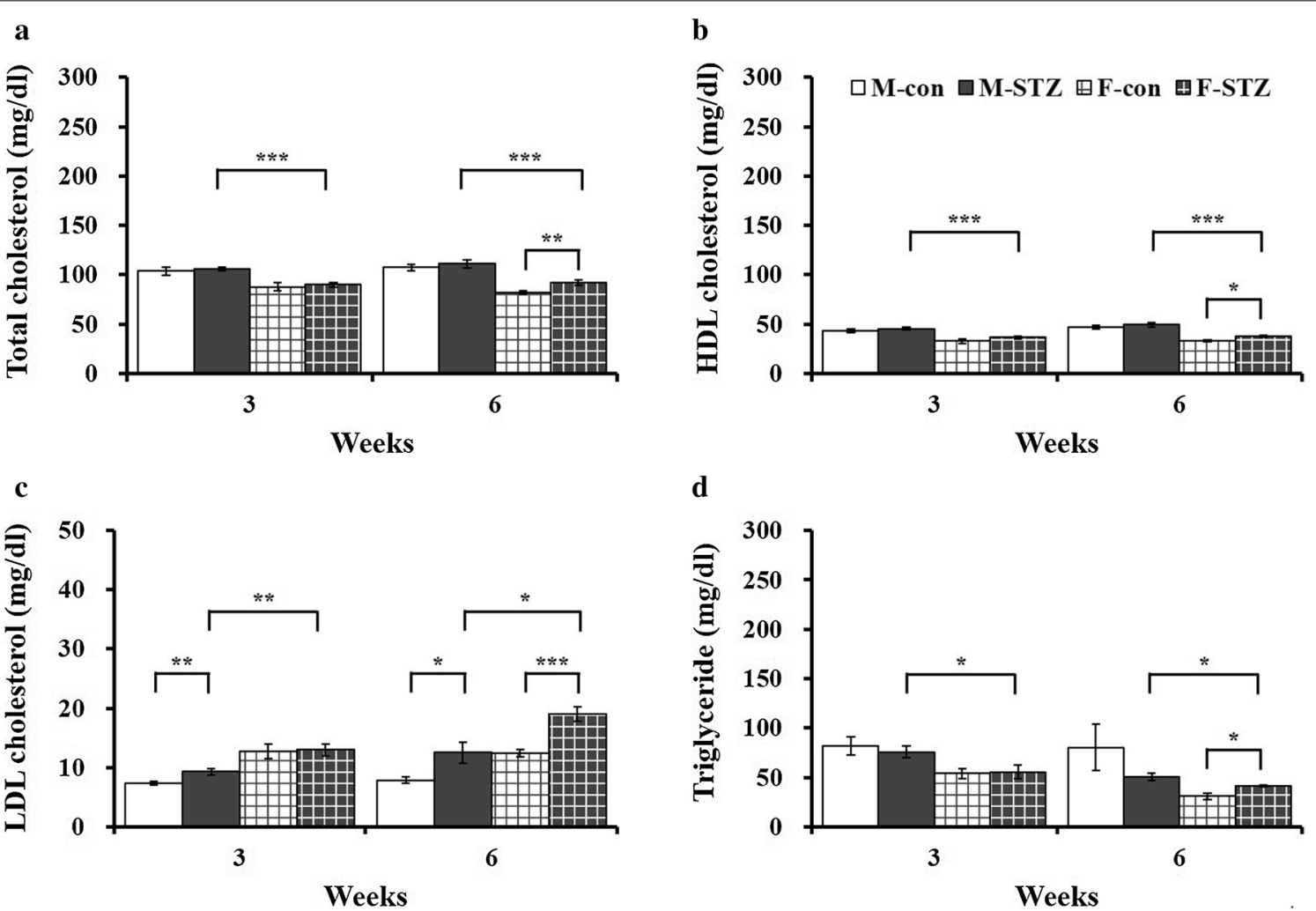

Fig. 4 Lipid profiles in streptozotocin (STZ)-induced diabetes mice at 3 and 6 weeks after STZ treatment. a Total cholesterol. b High-density lipoprotein cholesterol. c Low-density lipoprotein cholesterol. d Triglyceride. Lipid profiles in the STZ-induced female group were significantly higher than those in the female control group at 6 weeks after STZ treatment 
that in the F-con group at 6 weeks after STZ treatment (Fig. 4d). These results show that the lipid profiles of female mice by STZ treatment were lower than those of male mice.

\section{IR in the M-STZ group is more severe compared to that in the F-STZ group}

To assess $\beta$-cell function and IR after STZ treatment, HOMA-IR was calculated from fasting glucose and insulin concentrations. At 3 weeks after STZ treatment, steady-state $\beta$-cell functions (\%B) of the M-STZ and F-STZ groups were significantly lower than those of the M-con and F-con, groups, respectively (Fig. 5a). The insulin sensitivities (\%S) of the M-STZ and F-STZ groups were significantly lower than those of the M-con and F-con groups, respectively (Fig. 5b), simultaneously. Thus, the HOMA-IR values of the M-STZ and F-STZ groups were significantly different from those of the M-con and F-con groups, respectively (Fig. 5c). The $\% \mathrm{~B}, \% \mathrm{~S}$, and HOMA-IR in the M-STZ group were significantly worse than those in the F-STZ group, indicating that male mice are more sensitive to STZ toxicity than female mice at 3 weeks after STZ treatment.

At 6 weeks after STZ treatment, the \%B of the M-STZ and F-STZ groups were significantly lower than those of the M-con and F-con groups, respectively (Fig. 5a). The $\% \mathrm{~S}$ and HOMA-IR values of the M-STZ group were significantly lower than those of the M-con group. However, the $\% \mathrm{~S}$ and HOMA-IR value in the F-STZ group were not significantly different from those of the F-con group, indicating that IR was not observed in the F-STZ group at 6 weeks after STZ treatment (Fig. 5b, c).

\section{Discussion}

Diabetes mellitus is a disease with different glucose metabolisms according to sex. To elucidate this question in mice, the phenotypic differences by sex in STZinduced diabetic animals were analyzed. The FBG levels in the M-STZ group were higher by $35.9 \%$ and $74.5 \%$ than those in the M-con group at 3 and 6 weeks after STZ treatment. The FBG levels in the F-STZ group were higher by $10 \%$ and $25.8 \%$ than those in the F-con group at 3 and 6 weeks after STZ treatment. The FBG level in
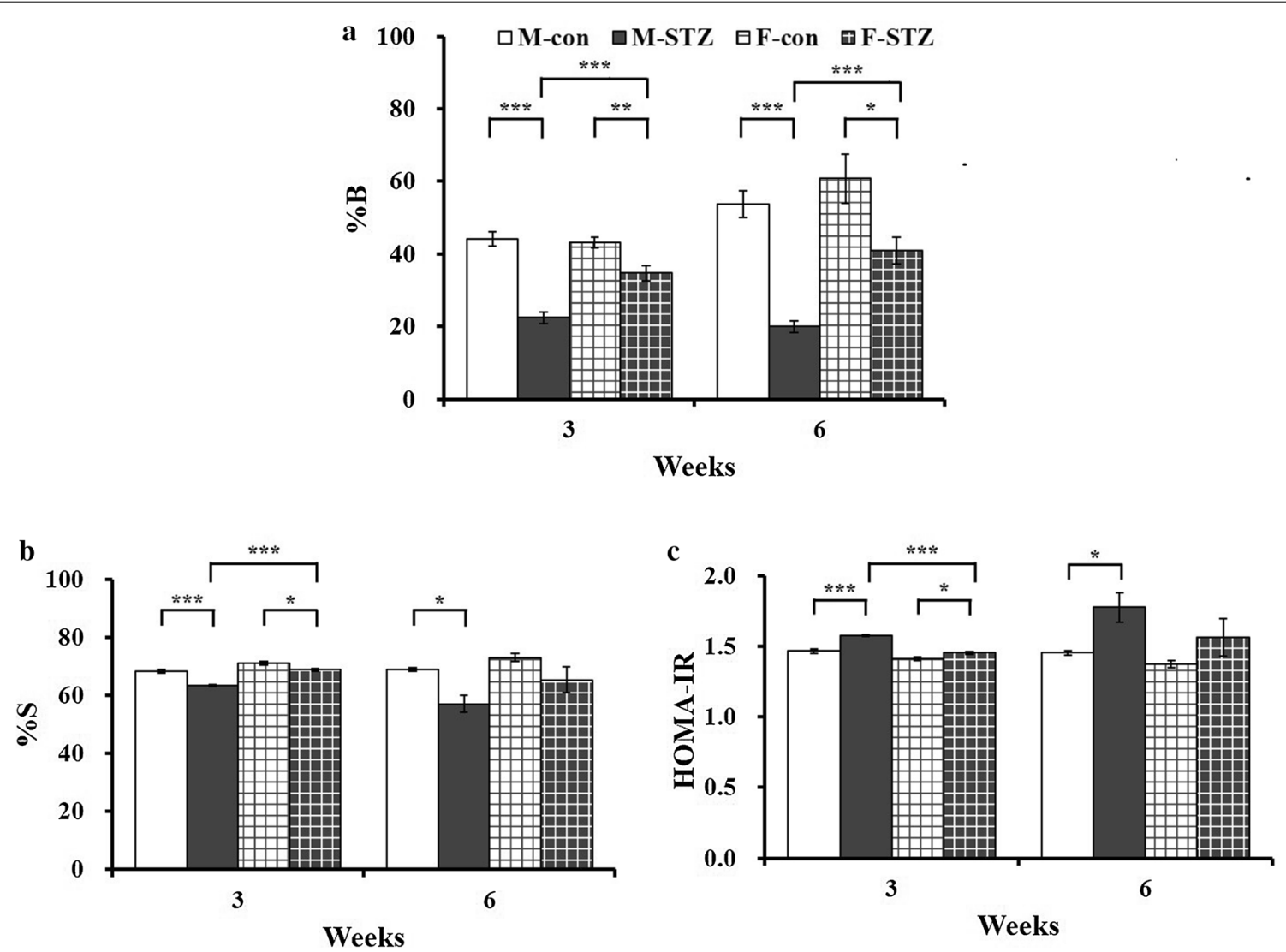

Fig. 5 Insulin resistance and $\beta$-cell function in streptozotocin (STZ)-induced diabetes mice at 3 and 6 weeks after STZ treatment. a Steady-state $\beta$-cell function. b Insulin sensitivity (\%S). c Homeostatic model assessment (HOMA)-insulin resistance (IR). There were no significant differences in $\% S$ and HOMA-IR between the STZ-induced female and female control groups at 6 weeks after STZ treatment 
the M-STZ group was significantly higher than that in the F-STZ group during the entire experimental period. The $\mathrm{HbA}_{1 \mathrm{C}}$ level in the M-STZ group was significantly higher than that in the M-con group. These results indicate that STZ is more toxic in the pancreatic islets of male mice compared to those in female mice. Ariza et al. [15] reported that blood glucose levels were higher in STZ-treated ICR males than those in STZ-treated ICR females until 23 weeks of age. Chandramouli et al. [12] also reported that hyperglycemia and mild diastolic dysfunction were more frequently observed in STZ-treated male mice compared to STZ-treated female mice. Taken together, the pancreatic islets in male mice are more sensitive to STZ compared to those in female mice at all ages, indicating that researchers must consider sex-specific differences when conducting glucose metabolism research.

In this study, glucose tolerance was observed in the M-STZ and F-STZ groups at 3 and 6 weeks after STZ treatment. Furthermore, glucose tolerance in the M-STZ group at 3 and 6 weeks after STZ treatment was more severe than that in the F-STZ group. In immunohistochemical analyses, the $\beta$-cell mass in the M-STZ group was significantly lower than that in the F-STZ group at 6 weeks after STZ treatment. The $\alpha$-cell mass and glucagon/insulin ratio in the M-STZ group were significantly higher than those in the M-con group. Thus, the glucagon/insulin ratio in the M-STZ group was significantly different from that of the F-STZ group at 6 weeks after STZ treatment. These results suggested that the pancreatic islets in the F-STZ group had been restored between 3 and 6 weeks after STZ treatment. Total cholesterol, HDL cholesterol, LDL cholesterol, and triglyceride levels were significantly higher in the F-STZ group compared with those in the F-con group at 6 weeks after STZ treatment. Moreover, LDL cholesterol level was significantly higher in the F-STZ group compared to that in the M-STZ group at 3 and 6 weeks. Carnevale Schianca et al. reported that type 2 diabetic women had worse plasma lipid composition compared to men [16]. Thus, these results indicate that hyperlipidemia develops differently between sexes. Values of HOMA-IR, an indicator of $\beta$-cell function and IR, significantly increased in the M-STZ and F-STZ groups at 3 weeks after STZ treatment. The HOMA-IR value in the F-STZ group was not significantly different from that in the F-con group, indicating that $\beta$-cell dysfunction and IR in the F-STZ group were restored at 6 weeks after STZ treatment.

Estrogens promote $\beta$-cell function by promoting misfolded proinsulin degradation in mice [17]. Premenopausal women have decreased estradiol levels and $47 \%$ increased risk of diabetes [18]. Surgical menopause (ovariectomy) women are also associated with an increased risk of diabetes [10]. Since estrogens might restore $\beta$-cell function in the F-STZ group, the FBG level in the F-STZ group was lower than that in the M-STZ group throughout the experimental periods. Furthermore, glucose tolerance in the F-STZ group was no longer observed at 6 weeks after STZ treatment. To confirm this hypothesis, women with severe estrogen deficiency (ovariectomy) should be considered when conducting a study, and the results should also be compared with women having normal estrogen concentration.

Androgen is an endogenous steroid hormone that develops and maintains male characteristics. Androgen is mainly synthesized in the testes, adrenal glands, and ovaries in women. Men are diagnosed with type 2 diabetes as a result of low testosterone levels [19]. Prostate cancer patients treated with androgen deprivation therapy (very low testosterone level) have increased diabetic risk and show hyperglycemia via decreased $\beta$-cell function $[20,21]$. Taken together, androgens have critical roles in glucose metabolism, obesity, and type 2 diabetes in men. To determine the roles of androgens in STZ-induced diabetic males, it is necessary to compare the experimental results between severe androgen deficiency (castration) and normal males.

\section{Acknowledgements \\ The authors thank MJ. Jung and YW. Park from Laboratory Animal Resource Center, Hallym University for the care of animals. The authors would like to thank Editage for English language editing.}

\section{Authors' contributions}

BK, YYK, PTN, and HM performed experiment. JGS designed experiments, analyzed data, and wrote the manuscript. All authors read and approved the final manuscript.

\section{Funding}

This work was supported by Basic Science Research Program (NRF-2016R1D1A2B02011858) and Mouse Phenotyping Project (KMPC:2014M3A9D5A01075129) of the Ministry of Science, ICT and Future Planning through the National Research Foundation of Korea.

\section{Availability of data and materials}

The datasets used and analyzed in this study are available from the corresponding author upon reasonable request.

\section{Competing interests}

The authors declare that there is no financial conflict of interests to publish these results.

\section{Author details}

1 Department of Medical Genetics, College of Medicine, Hallym University, 1, Hallymdaehak-gil, Chuncheon, Gangwon-do 24252, South Korea. ${ }^{2}$ Institute of Medical Science, College of Medicine, Hallym University, 1, Hallymdaehak-gil, Chuncheon, Gangwon-do 24252, South Korea.

Received: 26 June 2020 Accepted: 14 September 2020

Published online: 22 September 2020

\section{References}

1. Beery AK, Zucker I (2011) Sex bias in neuroscience and biomedical research. Neurosci Biobehav Rev 35(3):565-572 
2. Chen X, McClusky R, Chen J, Beaven SW, Tontonoz P, Arnold AP, Reue K, Beery AK, Zucker I (2012) The number of $x$ chromosomes causes sex differences in adiposity in mice. PLoS Genet 8(5):e1002709

3. Sorge RE, Martin LJ, Isbester KA, Sotocinal SG, Rosen S, Tuttle AH, Wieskopf JS, Acland EL, Dokova A, Kadoura B, Leger P, Mapplebeck JC, McPhail M, Delaney A, Wigerblad G, Schumann AP, Quinn T, Frasnelli J, Svensson Cl, Sternberg WF, Mogil JS (2014) Olfactory exposure to males, including men, causes stress and related analgesia in rodents. Nat Methods 6:629-632

4. Sorge RE, Mapplebeck JC, Rosen S, Beggs S, Taves S, Alexander JK, Martin LJ, Austin JS, Sotocinal SG, Chen D, Yang M, Shi XQ, Huang H, Pillon NJ, Bilan PJ, Tu Y, Klip A, Ji RR, Zhang J, Salter MW, Mogil JS (2015) Different immune cells mediate mechanical pain hypersensitivity in male and female mice. Nat Neurosci 18(8):1081-1083

5. Clayton JA, Collins FS (2014) Policy: NIH to balance sex in cell and animal studies. Nature 509(7500):282-283

6. Mauvais-Jarvis F (2018) Gender differences in glucose homeostasis and diabetes. Physiol Behav 187:20-23

7. Frias JP, Macaraeg GB, Ofrecio J, Yu JG, Olefsky JM, Kruszynska YT (2001) Decreased susceptibility to fatty acid-induced peripheral tissue insulin resistance in women. Diabetes 50(6):1344-1350

8. Brand JS, van der Schouw YT, Onland-Moret NC, Sharp SJ, Sharp SJ, Ong KK, Ong KK, Ong KK, Khaw KT, Khaw KT, Ardanaz E, Ardanaz E, Amiano P, Amiano P, Boeing $H$, Boeing $H$, Chirlaque MD, Clavel-Chapelon F, Crowe FL, de Lauzon-Guillain B, Duell EJ, Duell EJ, Fagherazzi G, Fagherazzi G, Franks PW, Franks PW, Grioni S, Grioni S, Groop LC, Groop LC, Groop LC, Groop LC, Kaaks R, Key TJ, Nilsson PM, Overvad K, Overvad K, Palli D, Palli D, Panico S, Panico S, Quirós JR, Rolandsson O, Sacerdote C, Sánchez MJ, Slimani N, Teucher B, Tjonneland A, Tumino R, Van der A, Feskens EJ, Langenberg C, Forouhi NG, Forouhi NG, Riboli E, Riboli E, Wareham NJ, Teucher B, Tjonneland A, InterAct Consortium., Inter Act Consortium. (2013) Age at menopause, reproductive life span, and type 2 diabetes risk: results from the EPIC-InterAct study. Diabetes Care 36:1012-1019

9. Shen L, Song L, Li H, Liu B, Zheng X, Zhang L, Yuan J, Liang Y, Wang Y (2017) Association between earlier age at natural menopause and risk of diabetes in middle-aged and older Chinese women: the DongfengTongji cohort study. Diabete Metab 43(3):345-350

10. Malacara JM, Huerta R, Rivera B, Esparza S, Fajardo ME (1997) Menopause in normal and uncomplicated NIDDM women: physical and emotional symptoms and hormone profile. Maturitas. 28(1):35-45

11. Keating NL, O'Malley AJ, Smith MR (2006) Diabetes and cardiovascular disease during androgen deprivation therapy for prostate cancer. J Clin Oncol 24(27):4448-4456
12. Chandramouli C, Reichelt ME, Curl CL, Varma U, Bienvenu LA, Koutsifeli P, Raaijmakers AJA, De Blasio MJ, Qin CX, Jenkins AJ, Ritchie RH, Mellor KM, Delbridge LMD (2018) Diastolic dysfunction is more apparent in STZinduced diabetic female mice, despite less pronounced hyperglycemia. Sci Rep. 8(1):2346

13. Ohta T, Katsuda Y, Miyajima K, Sasase T, Kimura S, Tong B, Yamada T (2014) Gender differences in metabolic disorders and related diseases in Spontaneously Diabetic Torii-Lepr (fa) rats. J Diabetes Res. 2004:841957

14. Kim B, Kim YY, Jung H, Nam H, Suh JG (2019) Delayed onset of obesity and glucose tolerance in interleukin 18 deficient mice by single housed condition. Appl Biol Chem. 62:1

15. Ariza L, Zaguirre M, García M, Blasco E, Rabanal RM, Bosch A, Otaegui PJ (2014) Hyperglycemia and hepatic tumors in ICR mice neonatally injected with streptozotocin. Lab Anim. 43(7):242-249

16. Carnevale Schianca GP, Fra GP, Colli E, Bigliocca M, Mella R, Scaglia E, Bartoli $E$ (2012) Sex differences in lipid profiles in relation to the progression of glucose abnormalities. J Diabetes. 4(1):95-101

17. Xu B, Allard C, Alvarez-Mercado Al, Fuselier T, Kim JH, Coons LA, Hewitt SC, Urano F, Korach KS, Levin ER, Arvan P, Floyd ZE, Mauvais-Jarvis F (2018) Estrogens promote misfolded proinsulin degradation to protect insulin production and delay diabetes. Cell Rep. 24(1):181-196

18. Park SK, Harlow SD, Zheng H, Karvonen-Gutierrez C, Thurston RC, Ruppert K, Janssen I, Randolph JF Jr (2017) Association between changes in oestradiol and follicle-stimulating hormone levels during the menopausal transition and risk of diabetes. Diabet Med 34(4):531-538

19. Zitzmann M (2009) Testosterone deficiency, insulin resistance and the metabolic syndrome. Nat Rev Endocrinol. 5(12):673-681

20. Corona G, Monami M, Rastrelli G, Aversa A, Sforza A, Lenzi A, Forti G, Mannucci E, Maggi M (2011) Type 2 diabetes mellitus and testosterone: a meta-analysis study. Int J Androl 34(6 Pt 1):528-540

21. Navarro G, Allard C, Xu W, Mauvais-Jarvis F (2015) The role of androgens in metabolism, obesity, and diabetes in males and females. Obesity 23(4):713-719

\section{Publisher's Note}

Springer Nature remains neutral with regard to jurisdictional claims in published maps and institutional affiliations.

\section{Submit your manuscript to a SpringerOpen ${ }^{\circ}$ journal and benefit from:}

- Convenient online submission

- Rigorous peer review

- Open access: articles freely available online

- High visibility within the field

- Retaining the copyright to your article

Submit your next manuscript at $\boldsymbol{\nabla}$ springeropen.com 